\title{
A General Approximation Method for a Kind of Convex Optimization Problems in Hilbert Spaces
}

\author{
Ming Tian and Li-Hua Huang \\ College of Science, Civil Aviation University of China, Tianjin 300300, China \\ Correspondence should be addressed to Ming Tian; tianming1963@126.com
}

Received 13 December 2013; Revised 26 March 2014; Accepted 1 April 2014; Published 17 April 2014

Academic Editor: Yongkun Li

Copyright (C) 2014 M. Tian and L.-H. Huang. This is an open access article distributed under the Creative Commons Attribution License, which permits unrestricted use, distribution, and reproduction in any medium, provided the original work is properly cited.

The constrained convex minimization problem is to find a point $x^{*}$ with the property that $x^{*} \in C$, and $h\left(x^{*}\right)=\min h(x), \forall x \in C$, where $C$ is a nonempty, closed, and convex subset of a real Hilbert space $H, h(x)$ is a real-valued convex function, and $h(x)$ is not Fréchet differentiable, but lower semicontinuous. In this paper, we discuss an iterative algorithm which is different from traditional gradient-projection algorithms. We firstly construct a bifunction $F_{1}(x, y)$ defined as $F_{1}(x, y)=h(y)-h(x)$. And we ensure the equilibrium problem for $F_{1}(x, y)$ equivalent to the above optimization problem. Then we use iterative methods for equilibrium problems to study the above optimization problem. Based on Jung's method (2011), we propose a general approximation method and prove the strong convergence of our algorithm to a solution of the above optimization problem. In addition, we apply the proposed iterative algorithm for finding a solution of the split feasibility problem and establish the strong convergence theorem. The results of this paper extend and improve some existing results.

\section{Introduction}

Let $H$ be a real Hilbert space with the inner product $\langle\cdot, \cdot\rangle$ and the induced norm $\|\cdot\|$. Let $C$ be a nonempty, closed, and convex subset of $H$. Recall that a mapping $T$ is nonexpansive (see [1]) if

$$
\|T x-T y\| \leq\|x-y\|, \quad \forall x, y \in C .
$$

We denote by $F(T)=\{x \in C: T x=x\}$ the set of fixed points of $T$. Recall that a self-mapping $f$ on $H$ is a contraction if there exists a constant $\alpha \in(0,1)$ such that

$$
\|f(x)-f(y)\| \leq \alpha\|x-y\|, \quad \forall x, y \in H .
$$

Consider the following constrained convex minimization problem:

$$
\min _{x \in C} h(x)
$$

where $h: C \rightarrow R$ is a real-valued convex function. Assume that the constrained convex minimization problem (3) is solvable, and let $S$ denote the set of solutions of (3). Assume that $h(x)$ is lower semicontinuous. Let $F_{1}$ be a bifunction from $C \times C$ to $\mathbb{R}$ defined by $F_{1}(x, y)=h(y)-h(x)$. It is easy to see that $S=\operatorname{EP}\left(F_{1}\right)$, where $\operatorname{EP}\left(F_{1}\right)$ denotes the set of solutions of equilibrium problem to find $x \in C$ such that

$$
F_{1}(x, y) \geq 0, \quad \forall y \in C .
$$

Hence, the optimization problem (3) is equivalent to the equilibrium problem (4).

For solving the convex optimization problem (3), Su and $\mathrm{Li}$ [2] introduced the following iterative scheme in a Hilbert space: $x_{1} \in H$ and

$$
\begin{gathered}
h(y)-h\left(u_{n}\right)+\frac{1}{r_{n}}\left\langle y-u_{n}, u_{n}-x_{n}\right\rangle \geq 0, \quad \forall y \in C, \\
x_{n+1}=\alpha_{n} f\left(x_{n}\right)+\gamma_{n} u_{n}+\beta_{n} x_{n},
\end{gathered}
$$

for all $n \in \mathbb{N}$, where $u_{n}=T_{r_{n}} x_{n},\left\{\alpha_{n}\right\},\left\{\beta_{n}\right\},\left\{\gamma_{n}\right\} \subset(0,1), \alpha_{n}+$ $\beta_{n}+\gamma_{n}=1$, and $\left\{r_{n}\right\} \subset(0, \infty)$. Under appropriate conditions, they proved that the sequence $\left\{x_{n}\right\}$ generated by (5) converges strongly to a point $z=P_{S} f(z)$ of optimization problem (3). 
In 2010, combining Yamada's method [3] and Marino and Xu's method [4], Tian [5] proposed a general iterative algorithm:

$$
x_{n+1}=\alpha_{n} \gamma f\left(x_{n}\right)+\left(I-\alpha_{n} \mu F\right) T\left(x_{n}\right),
$$

where $f: H \rightarrow H$ is a contraction with coefficient $0<$ $\alpha<1$ and $F: H \rightarrow H$ is a $k$-Lipschitzian and $\eta$-strongly monotone operator with $k>0, \eta>0$. Let $0<\mu<2 \eta / k^{2}$, $0<\gamma<\mu\left(\eta-\left(\mu k^{2} / 2\right)\right) / \alpha=\tau / \alpha$. It is proved that the sequence $\left\{x_{n}\right\}_{n=0}^{\infty}$ generated by (6) converges strongly to a fixed point $\tilde{x} \in \operatorname{Fix}(T)$, which solves the variational inequality

$$
\langle(\gamma f-\mu F) \tilde{x}, x-\tilde{x}\rangle \leq 0, \quad \forall x \in \operatorname{Fix}(T) .
$$

In 2011, Jung [6] introduced the following general iterative scheme for $k$-strictly pseudocontractive mapping $T$ :

$$
x_{n+1}=\alpha_{n} \gamma f\left(x_{n}\right)+\left(\left(1-\beta_{n}\right) I-\alpha_{n} \mu F\right) P_{C} S^{\prime}\left(x_{n}\right)+\beta_{n} x_{n},
$$

where $S^{\prime}: C \rightarrow H$ is a mapping defined by $S^{\prime} x=k x+$ $(1-k) T x$ and $P_{C}$ is the metric projection of $H$ onto $C$. Under appropriate conditions, he established the strong convergence of the sequence $\left\{x_{n}\right\}$ generated by (8) to a fixed point of $T$, which is a solution of the variational inequality (7).

In this paper, motivated and inspired by the above results, we introduce a general iterative method: $x_{1} \in H$ and

$$
\begin{gathered}
h(y)-h\left(u_{n}\right)+\frac{1}{r_{n}}\left\langle y-u_{n}, u_{n}-x_{n}\right\rangle \geq 0, \quad \forall y \in C, \\
x_{n+1}=\alpha_{n} \gamma f\left(x_{n}\right)+\left(\left(1-\beta_{n}\right) I-\alpha_{n} \mu F\right) u_{n}+\beta_{n} x_{n}, \quad n \in \mathbb{N},
\end{gathered}
$$

for solving the optimization problem (3), where $u_{n}=T_{r_{n}} x_{n}$. Under appropriate conditions, it is proved that the sequence $\left\{x_{n}\right\}$ generated by (9) converges strongly to a point $z \in S$ which solves the variational inequality

$$
\langle(\mu F-\gamma f) z, z-x\rangle \leq 0, \quad \forall x \in S
$$

Furthermore, by using the above result we studied the split feasibility problem and obtained the iterative algorithm for solving the split feasibility problem.

\section{Preliminaries}

In this section we introduce some useful definitions and lemmas which will be used in the proofs for the main results in the next section.

Monotone operators are very useful in the convergence analysis.

Definition 1 (see [7] for comprehensive theory of monotone operators). Let $A: H \rightarrow H$ be an operator.

(i) $A$ is monotone, if and only if

$$
\langle x-y, A x-A y\rangle \geq 0, \quad \forall x, y \in H \text {. }
$$

(ii) $A$ is said to be $\eta$-strongly monotone, if there exists a positive constant $\eta$ such that

$$
\langle x-y, A x-A y\rangle \geq \eta\|x-y\|^{2}, \quad \forall x, y \in H .
$$

(iii) $A$ is said to be $v$-inverse strongly monotone ( $v$-ism), if there exists a positive constant $v$ such that

$$
\langle x-y, A x-A y\rangle \geq v\|A x-A y\|^{2}, \quad \forall x, y \in H .
$$

It is known that inverse strongly monotone operators have been studied widely (see [7-9]) and applied to solve practical problems in various fields, for instance, in traffic assignment problems (see $[10,11]$ ).

Lemma 2 (see [5]). Let $H$ be a Hilbert space, $f: H \rightarrow H a$ contraction with a coefficient $0<\rho<1$, and $F: H \rightarrow H a$ $k$-Lipschitz continuous and $\eta$-strongly monotone operator with constants $k>0, \eta>0$. Then, for $0<\gamma<\mu \eta / \rho$,

$$
\begin{array}{r}
\langle x-y,(\mu F-\gamma f) x-(\mu F-\gamma f) y\rangle \geq(\mu \eta-\gamma \rho)\|x-y\|^{2}, \\
\forall x, y \in H .
\end{array}
$$

That is, $\mu F-\gamma f$ is strongly monotone with a coefficient $\mu \eta-\gamma \rho$.

Recall the metric (nearest point) projection $P_{C}$ from a real Hilbert space $H$ to a closed and convex subset $C$ of $H$ which is defined as follows: given $x \in H, P_{C} x$ is the unique point in $C$ with the property

$$
\left\|x-P_{C} x\right\|=\inf \{\|x-y\|: y \in C\} .
$$

$P_{C}$ is characterized as follows.

Lemma 3. Let $C$ be a closed and convex subset of a real Hilbert space $H$. Given $x \in H$ and $y \in C$, then $y=P_{C} x$ if and only if the following inequality holds:

$$
\langle x-y, y-z\rangle \geq 0, \quad \forall z \in C .
$$

For solving the equilibrium problem for a bifunction $F_{1}$ : $C \times C \rightarrow \mathbb{R}$, let us assume that $F_{1}$ satisfies the following conditions:

(A1) $F_{1}(x, x)=0$ for all $x \in C$;

(A2) $F_{1}$ is monotone; that is, $F_{1}(x, y)+F_{1}(y, x) \leq 0$ for all $x, y \in C$;

(A3) for each $x, y, z \in C, \lim \sup _{t \rightarrow 0^{+}} F_{1}(t z+(1-t) x, y) \leq$ $F_{1}(x, y)$;

(A4) for each $x \in C, y \mapsto F_{1}(x, y)$ is convex and lower semicontinuous.

Lemma 4 (see [12]). Let $C$ be a nonempty, closed, and convex subset of $H$ and let $F_{1}$ be a bifunction of $C \times C$ into $\mathbb{R}$ satisfying (A1)-(A4). Let $r>0$ and $x \in H$. Then, there exists $z \in C$ such that

$$
F_{1}(z, y)+\frac{1}{r}\langle y-z, z-x\rangle \geq 0 \quad \forall y \in C .
$$


Lemma 5 (see [13]). Assume that $F_{1}: C \times C \rightarrow \mathbb{R}$ satisfies $(A 1)-(A 4)$. For $r>0$ and $x \in H$, define a mapping $T_{r}: H \rightarrow$ $C$ as follows:

$$
T_{r}(x)=\left\{z \in C: F_{1}(z, y)+\frac{1}{r}\langle y-z, z-x\rangle \geq 0, \forall y \in C\right\} .
$$

Then, the following hold.

(1) $T_{r}$ is single-valued;

(2) $T_{r}$ is firmly nonexpansive; that is, $\left\|T_{r} x-T_{r} y\right\|^{2} \leq$ $\left\langle T_{r} x-T_{r} y, x-y\right\rangle$ for any $x, y \in H$;

(3) $F\left(T_{r}\right)=E P\left(F_{1}\right)$;

(4) $E P\left(F_{1}\right)$ is closed and convex.

Lemma 6 (see [14]). Assume that $\left\{a_{n}\right\}_{n=0}^{\infty}$ is a sequence of nonnegative real numbers such that

$$
a_{n+1} \leq\left(1-\gamma_{n}\right) a_{n}+\gamma_{n} \delta_{n}, \quad n \geq 0,
$$

where $\left\{\gamma_{n}\right\}_{n=0}^{\infty}$ is a sequence in $(0,1)$ and $\left\{\delta_{n}\right\}_{n=0}^{\infty}$ is a sequence in $\mathbb{R}$ such that

(i) $\sum_{n=0}^{\infty} \gamma_{n}=\infty$;

(ii) either $\lim \sup _{n \rightarrow \infty} \delta_{n} \leq 0$ or $\sum_{n=0}^{\infty} \gamma_{n}\left|\delta_{n}\right|<\infty$.

Then $\lim _{n \rightarrow \infty} a_{n}=0$.

We adopt the following notation:

(i) $x_{n} \rightarrow x$ means that $x_{n} \rightarrow x$ strongly;

(ii) $x_{n} \rightarrow x$ means that $x_{n} \rightarrow x$ weakly.

\section{Main Results}

Recall that throughout this paper we always use $S$ to denote the solution set of the constrained convex minimization problem (3).

Let $H$ be a real Hilbert space and let $C$ be a nonempty, closed, and convex subset of $H$. Let $h: C \rightarrow \mathbb{R}$ be a real-valued convex function. Assume that $h(x)$ is lower semicontinuous. Let $f: H \rightarrow H$ be a contraction with a coefficient $0<\rho<1$, and let $F: C \rightarrow H$ be a $k$-Lipschitzian and $\eta$-strongly monotone operator with constants $k>0$, $\eta>0$. Suppose that $0<\mu<2 \eta / k^{2}, 0<\gamma<\tau / \rho$, where $\tau=\mu \eta-\left(\mu^{2} k^{2} / 2\right)$.

Next, we study the following iterative method. For a given arbitrary initial guess $x_{1} \in H$, the sequence $\left\{x_{n}\right\}_{n=0}^{\infty}$ is generated by the following recursive formula:

$$
\begin{gathered}
h(y)-h\left(u_{n}\right)+\frac{1}{r_{n}}\left\langle y-u_{n}, u_{n}-x_{n}\right\rangle \geq 0, \quad \forall y \in C, \\
x_{n+1}=\alpha_{n} \gamma f\left(x_{n}\right)+\left(\left(1-\beta_{n}\right) I-\alpha_{n} \mu F\right) u_{n}+\beta_{n} x_{n}, \quad n \in \mathbb{N},
\end{gathered}
$$

where $u_{n}=T_{r_{n}} x_{n}$ and $T_{r_{n}}$ is a mapping defined as in Lemma 5.

In order to prove the convergence, we also need the following proposition.
Proposition 7. Let $H$ be a real Hilbert space and let $F: H \rightarrow$ $H$ be a $k$-Lipschitzian and $\eta$-strongly monotone operator with constants $k>0, \eta>0$. Suppose that $0<\mu<2 \eta / k^{2}$, and $\left\{\alpha_{n}\right\}$, $\left\{\beta_{n}\right\} \subset(0,1)$ satisfy $0<\alpha_{n}<\min \left\{\left(1-\beta_{n}\right) / 2 \tau, 1-\beta_{n}\right\}$. Then, the mapping $G(x)=\left(\left(1-\beta_{n}\right) I-\alpha_{n} \mu F\right)(x)$ is a contraction with a contractive constant $1-\beta_{n}-\alpha_{n} \tau$, where $\tau=\mu \eta-\left(\mu^{2} k^{2} / 2\right)$.

Proof. Taking $x, y \in H$, we have

$$
\begin{aligned}
&\|G(x)-G(y)\|^{2} \\
&=\left\|\left(\left(1-\beta_{n}\right) I-\alpha_{n} \mu F\right)(x)-\left(\left(1-\beta_{n}\right) I-\alpha_{n} \mu F\right)(y)\right\|^{2} \\
&=\left(1-\beta_{n}\right)^{2}\|x-y\|^{2}-2 \alpha_{n} \mu\left(1-\beta_{n}\right)\langle x-y, F(x)-F(y)\rangle \\
&+\alpha_{n}^{2} \mu^{2}\|F(x)-F(y)\|^{2} \\
& \leq {\left[\left(1-\beta_{n}\right)^{2}-2 \alpha_{n} \mu\left(1-\beta_{n}\right) \eta+\alpha_{n}^{2} \mu^{2} k^{2}\right]\|x-y\|^{2} } \\
& \leq\left(1-\beta_{n}\right)^{2}\left[1-\frac{2 \alpha_{n} \mu \eta}{1-\beta_{n}}+\frac{\alpha_{n} \mu^{2} k^{2}}{1-\beta_{n}}\right]\|x-y\|^{2} .
\end{aligned}
$$

Thus,

$$
\begin{aligned}
& \|G(x)-G(y)\| \\
& \quad \leq\left(1-\beta_{n}\right) \sqrt{1-\alpha_{n}\left(\frac{2 \mu \eta}{1-\beta_{n}}-\frac{\mu^{2} k^{2}}{1-\beta_{n}}\right)}\|x-y\| \\
& \quad \leq\left(1-\beta_{n}\right)\left[1-\alpha_{n}\left(\frac{\mu \eta}{1-\beta_{n}}-\frac{\mu^{2} k^{2}}{2\left(1-\beta_{n}\right)}\right)\right]\|x-y\| \\
& \quad=\left(1-\beta_{n}-\alpha_{n} \tau\right)\|x-y\|,
\end{aligned}
$$

where $\tau=\mu \eta-\left(\mu^{2} k^{2} / 2\right)$.

Theorem 8. Let $H$ be a real Hilbert space and let $C$ be a nonempty, closed, and convex subset of $H$. Let $h: C \rightarrow \mathbb{R}$ be a real-valued convex function. Assume that $h(x)$ is lower semicontinuous. Let $f: H \rightarrow H$ be a contraction with a coefficient $0<\rho<1$ and let $F: C \rightarrow H$ be a $k$ Lipschitzian and $\eta$-strongly monotone operator with constants $k>0, \eta>0$. Suppose that $0<\mu<2 \eta / k^{2}, 0<\gamma<\tau / \rho$, where $\tau=\mu \eta-\left(\mu^{2} k^{2} / 2\right)$. Suppose that the optimization problem (3) is consistent and let $S$ denote its solution set. Let $\left\{x_{n}\right\}_{n=0}^{\infty}$ be generated by $x_{1} \in H$ and

$$
\begin{gathered}
h(y)-h\left(u_{n}\right)+\frac{1}{r_{n}}\left\langle y-u_{n}, u_{n}-x_{n}\right\rangle \geq 0, \quad \forall y \in C, \\
x_{n+1}=\alpha_{n} \gamma f\left(x_{n}\right)+\left(\left(1-\beta_{n}\right) I-\alpha_{n} \mu F\right) u_{n}+\beta_{n} x_{n}, \quad n \in \mathbb{N},
\end{gathered}
$$

where $u_{n}=T_{r_{n}} x_{n}$ and $T_{r_{n}}$ is a mapping defined as in Lemma 5. $\left\{\alpha_{n}\right\},\left\{\beta_{n}\right\} \subset(0,1),\left\{r_{n}\right\} \subset(0, \infty)$ satisfy the following conditions:

(C1) $\lim _{n \rightarrow \infty} \alpha_{n}=0$ and $\sum_{n=1}^{\infty} \alpha_{n}=\infty$;

(C2) $\sum_{n=1}^{\infty}\left|\alpha_{n+1}-\alpha_{n}\right|<\infty$; 
(C3) $\limsup _{n \rightarrow \infty} \beta_{n}<1$;

(C4) $\sum_{n=1}^{\infty}\left|\beta_{n+1}-\beta_{n}\right|<\infty$;

(C5) $\liminf _{n \rightarrow \infty} r_{n}>0$ and $\sum_{n=1}^{\infty}\left|r_{n+1}-r_{n}\right|<\infty$.

Then the sequence $\left\{x_{n}\right\}$ generated by (23) converges strongly to a point $z \in S$, which solves the variational inequality

$$
\langle(\mu F-\gamma f) z, z-x\rangle \leq 0, \quad \forall x \in S
$$

Proof. Let $F_{1}$ be a bifunction from $C \times C$ to $\mathbb{R}$ defined by $F_{1}(x, y)=h(y)-h(x)$. We consider the following equilibrium problem, that is, to find $x \in C$, such that

$$
F_{1}(x, y) \geq 0, \quad \forall y \in C
$$

It is obvious that $\operatorname{EP}\left(F_{1}\right)=S$, where $\operatorname{EP}\left(F_{1}\right)$ denotes the set of solutions of equilibrium problem (25). In addition, it is easy to see that $F_{1}(x, y)$ satisfies the conditions $(A 1)-(A 4)$ in Section 2. Then, the iterative method (23) is equivalent to $x_{1} \in H$ and

$$
\begin{gathered}
F_{1}\left(u_{n}, y\right)+\frac{1}{r_{n}}\left\langle y-u_{n}, u_{n}-x_{n}\right\rangle \geq 0, \quad \forall y \in C, \\
x_{n+1}=\alpha_{n} \gamma f\left(x_{n}\right)+\left(\left(1-\beta_{n}\right) I-\alpha_{n} \mu F\right) u_{n}+\beta_{n} x_{n}, \quad n \in \mathbb{N},
\end{gathered}
$$

where $u_{n}=T_{r_{n}} x_{n}$.

It is easy to see the uniqueness of a solution of variational inequality (24). By Lemma $2, \mu F-\gamma f$ is strongly monotone, so variational inequality (24) has only one solution. Below, we use $z \in S$ to denote the unique solution of (24). Since $z \in S$ solves variational inequality (24), then (24) can be written as

$$
\langle(I-\mu F+\gamma f) z-z, z-x\rangle \geq 0, \quad \forall x \in S .
$$

So in terms of Lemma 3, it is equivalent to the following fixedpoint equation

$$
z=P_{S}(I-\mu F+\gamma f) z=P_{\mathrm{EP}\left(F_{1}\right)}(I-\mu F+\gamma f) z .
$$

Now, we show that $\left\{x_{n}\right\}$ is bounded. Indeed, picking $p \in S=$ $\operatorname{EP}\left(F_{1}\right)$, since $u_{n}=T_{r_{n}} x_{n}$, by Lemma 5 , we know that

$$
\left\|u_{n}-p\right\|=\left\|T_{r_{n}} x_{n}-T_{r_{n}} p\right\| \leq\left\|x_{n}-p\right\|, \quad \forall n \geq 1
$$

From Propositions 7 and (29), we derive that

$$
\begin{aligned}
& \left\|x_{n+1}-p\right\| \\
& =\left\|\alpha_{n} \gamma f\left(x_{n}\right)+\left(\left(1-\beta_{n}\right) I-\alpha_{n} \mu F\right) u_{n}+\beta_{n} x_{n}-p\right\| \\
& \leq \alpha_{n}\left\|\gamma f\left(x_{n}\right)-\mu F(p)\right\| \\
& \quad+\left\|\left(\left(1-\beta_{n}\right) I-\alpha_{n} \mu F\right) u_{n}-\left(\left(1-\beta_{n}\right) I-\alpha_{n} \mu F\right) p\right\| \\
& \quad+\beta_{n}\left\|x_{n}-p\right\|
\end{aligned}
$$

$$
\begin{aligned}
\leq & \alpha_{n} \gamma\left\|f\left(x_{n}\right)-f(p)\right\|+\alpha_{n}\|\gamma f(p)-\mu F(p)\| \\
& +\left(1-\beta_{n}-\alpha_{n} \tau\right)\left\|u_{n}-p\right\|+\beta_{n}\left\|x_{n}-p\right\| \\
\leq & \alpha_{n} \gamma \rho\left\|x_{n}-p\right\|+\alpha_{n}\|\gamma f(p)-\mu F(p)\| \\
& +\left(1-\beta_{n}-\alpha_{n} \tau\right)\left\|u_{n}-p\right\|+\beta_{n}\left\|x_{n}-p\right\| \\
\leq & \left(1-\alpha_{n}(\tau-\gamma \rho)\right)\left\|x_{n}-p\right\|+\alpha_{n}\|\gamma f(p)-\mu F(p)\| \\
= & \left(1-\alpha_{n}(\tau-\gamma \rho)\right)\left\|x_{n}-p\right\| \\
& +\alpha_{n}(\tau-\gamma \rho) \frac{\|\gamma f(p)-\mu F(p)\|}{\tau-\gamma \rho} \\
\leq & \max \left\{\left\|x_{n}-p\right\|, \frac{\|\gamma f(p)-\mu F(p)\|}{\tau-\gamma \rho}\right\} .
\end{aligned}
$$

By induction, we have

$$
\left\|x_{n}-p\right\| \leq \max \left\{\left\|x_{0}-p\right\|, \frac{\|\gamma f(p)-\mu F(p)\|}{\tau-\gamma \rho}\right\} .
$$

Hence $\left\{x_{n}\right\}$ is bounded. From (29), we also derive that $\left\{u_{n}\right\}$ is bounded.

Next, we show that $\left\|x_{n+1}-x_{n}\right\| \rightarrow 0$. Consider

$$
\begin{aligned}
& \left\|x_{n+1}-x_{n}\right\| \\
& =\|\left[\alpha_{n} \gamma f\left(x_{n}\right)+\left(\left(1-\beta_{n}\right) I-\alpha_{n} \mu F\right) u_{n}+\beta_{n} x_{n}\right] \\
& -\left[\alpha_{n-1} \gamma f\left(x_{n-1}\right)\right. \\
& \left.+\left(\left(1-\beta_{n-1}\right) I-\alpha_{n-1} \mu F\right) u_{n-1}+\beta_{n-1} x_{n-1}\right] \| \\
& \leq \alpha_{n} \gamma\left\|f\left(x_{n}\right)-f\left(x_{n-1}\right)\right\|+\left|\alpha_{n}-\alpha_{n-1}\right| \gamma\left\|f\left(x_{n-1}\right)\right\| \\
& +\left\|\left(\left(1-\beta_{n}\right) I-\alpha_{n} \mu F\right) u_{n}-\left(\left(1-\beta_{n}\right) I-\alpha_{n} \mu F\right) u_{n-1}\right\| \\
& +\left\|\left(\beta_{n-1}-\beta_{n}\right) u_{n-1}+\left(\alpha_{n-1}-\alpha_{n}\right) \mu F\left(u_{n-1}\right)\right\| \\
& +\left|\beta_{n}-\beta_{n-1}\right|\left\|x_{n-1}\right\|+\beta_{n}\left\|x_{n}-x_{n-1}\right\| \\
& \leq \alpha_{n} \gamma \rho\left\|x_{n}-x_{n-1}\right\|+\left|\alpha_{n}-\alpha_{n-1}\right| \gamma\left\|f\left(x_{n-1}\right)\right\| \\
& +\left(1-\beta_{n}-\alpha_{n} \tau\right)\left\|u_{n}-u_{n-1}\right\|+\left|\beta_{n}-\beta_{n-1}\right|\left\|u_{n-1}\right\| \\
& +\left|\alpha_{n}-\alpha_{n-1}\right|\left\|\mu F\left(u_{n-1}\right)\right\| \\
& +\left|\beta_{n}-\beta_{n-1}\right|\left\|x_{n-1}\right\|+\beta_{n}\left\|x_{n}-x_{n-1}\right\| \\
& =\left(\alpha_{n} \gamma \rho+\beta_{n}\right)\left\|x_{n}-x_{n-1}\right\| \\
& +\left|\alpha_{n}-\alpha_{n-1}\right|\left(\gamma\left\|f\left(x_{n-1}\right)\right\|+\left\|\mu F\left(u_{n-1}\right)\right\|\right) \\
& +\left(1-\beta_{n}-\alpha_{n} \tau\right)\left\|u_{n}-u_{n-1}\right\| \\
& +\left|\beta_{n}-\beta_{n-1}\right|\left(\left\|u_{n-1}\right\|+\left\|x_{n-1}\right\|\right) .
\end{aligned}
$$


From $u_{n+1}=T_{r_{n+1}} x_{n+1}$ and $u_{n}=T_{r_{n}} x_{n}$, we note that

$$
F_{1}\left(u_{n+1}, y\right)+\frac{1}{r_{n+1}}\left\langle y-u_{n+1}, u_{n+1}-x_{n+1}\right\rangle \geq 0, \quad \forall y \in C,
$$

$$
F_{1}\left(u_{n}, y\right)+\frac{1}{r_{n}}\left\langle y-u_{n}, u_{n}-x_{n}\right\rangle \geq 0, \quad \forall y \in C .
$$

Putting $y=u_{n}$ in (33) and $y=u_{n+1}$ in (34), we have

$$
\begin{gathered}
F_{1}\left(u_{n+1}, u_{n}\right)+\frac{1}{r_{n+1}}\left\langle u_{n}-u_{n+1}, u_{n+1}-x_{n+1}\right\rangle \geq 0, \\
F_{1}\left(u_{n}, u_{n+1}\right)+\frac{1}{r_{n}}\left\langle u_{n+1}-u_{n}, u_{n}-x_{n}\right\rangle \geq 0 .
\end{gathered}
$$

So, from (A2), we have

$$
\left\langle u_{n+1}-u_{n}, \frac{u_{n}-x_{n}}{r_{n}}-\frac{u_{n+1}-x_{n+1}}{r_{n+1}}\right\rangle \geq 0,
$$

and hence

$$
\left\langle u_{n+1}-u_{n}, u_{n}-u_{n+1}+u_{n+1}-x_{n}-\frac{r_{n}}{r_{n+1}}\left(u_{n+1}-x_{n+1}\right)\right\rangle
$$

$\geq 0$.

Since liminf $\operatorname{in}_{n \rightarrow \infty} r_{n}>0$, without loss of generality, let us assume that there exists a real number $a$ such that $r_{n}>a>0$ for all $n \in \mathbb{N}$. Thus, we have

$$
\begin{aligned}
& \left\|u_{n+1}-u_{n}\right\|^{2} \\
& \quad \leq\left\langle u_{n+1}-u_{n}, x_{n+1}-x_{n}+\left(1-\frac{r_{n}}{r_{n+1}}\right)\left(u_{n+1}-x_{n+1}\right)\right\rangle \\
& \quad \leq\left\|u_{n+1}-u_{n}\right\|\left\{\left\|x_{n+1}-x_{n}\right\|+\left|1-\frac{r_{n}}{r_{n+1}}\right|\left\|u_{n+1}-x_{n+1}\right\|\right\} ;
\end{aligned}
$$

thus,

$$
\left\|u_{n+1}-u_{n}\right\| \leq\left\|x_{n+1}-x_{n}\right\|+\frac{1}{a}\left|r_{n+1}-r_{n}\right| M_{1},
$$

where $M_{1}=\sup \left\{\left\|u_{n}-x_{n}\right\|: n \in \mathbb{N}\right\}$.

From (32) and (39), we obtain

$$
\begin{aligned}
& \left\|x_{n+1}-x_{n}\right\| \\
& \leq\left(\alpha_{n} \gamma \rho+\beta_{n}\right)\left\|x_{n}-x_{n-1}\right\| \\
& \quad+\left|\alpha_{n}-\alpha_{n-1}\right|\left(\gamma\left\|f\left(x_{n-1}\right)\right\|+\left\|\mu F\left(u_{n-1}\right)\right\|\right) \\
& \quad+\left(1-\beta_{n}-\alpha_{n} \tau\right)\left(\left\|x_{n}-x_{n-1}\right\|+\frac{1}{a}\left|r_{n}-r_{n-1}\right| M_{1}\right) \\
& \quad+\left|\beta_{n}-\beta_{n-1}\right|\left(\left\|u_{n-1}\right\|+\left\|x_{n-1}\right\|\right) \\
& \leq \\
& \quad\left(1-\alpha_{n} \tau+\alpha_{n} \gamma \rho\right)\left\|x_{n}-x_{n-1}\right\| \\
& \quad+\left|\alpha_{n}-\alpha_{n-1}\right|\left(\gamma\left\|f\left(x_{n-1}\right)\right\|+\left\|\mu F\left(u_{n-1}\right)\right\|\right) \\
& \quad+\frac{M_{1}}{a}\left|r_{n}-r_{n-1}\right|+\left|\beta_{n}-\beta_{n-1}\right|\left(\left\|u_{n-1}\right\|+\left\|x_{n-1}\right\|\right)
\end{aligned}
$$

$$
\begin{aligned}
\leq & \left(1-\alpha_{n}(\tau-\gamma \rho)\right)\left\|x_{n}-x_{n-1}\right\| \\
& +M_{2}\left(\left|\alpha_{n}-\alpha_{n-1}\right|+\left|r_{n}-r_{n-1}\right|+\left|\beta_{n}-\beta_{n-1}\right|\right),
\end{aligned}
$$

where $M_{2} \geq \max \left\{\gamma\left\|f\left(x_{n-1}\right)\right\|+\left\|\mu F\left(u_{n-1}\right)\right\|, M_{1} / a,\left\|u_{n-1}\right\|+\right.$ $\left.\left\|x_{n-1}\right\|\right\}$, for all $n \in \mathbb{N}$. Hence, by Lemma 6 , we have

$$
\lim _{n \rightarrow \infty}\left\|x_{n+1}-x_{n}\right\|=0 .
$$

Then, from (39), (41), and $\left|r_{n+1}-r_{n}\right| \rightarrow 0$, we have

$$
\lim _{n \rightarrow \infty}\left\|u_{n+1}-u_{n}\right\|=0 \text {. }
$$

Next, we show that $\left\|x_{n}-u_{n}\right\| \rightarrow 0$.

Indeed, for any $p \in \operatorname{EP}\left(F_{1}\right)$, by Lemma 5 , we have

$$
\begin{aligned}
\left\|u_{n}-p\right\|^{2} & =\left\|T_{r_{n}} x_{n}-T_{r_{n}} p\right\|^{2} \\
& \leq\left\langle x_{n}-p, u_{n}-p\right\rangle \\
& =\frac{1}{2}\left(\left\|x_{n}-p\right\|^{2}+\left\|u_{n}-p\right\|^{2}-\left\|u_{n}-x_{n}\right\|^{2}\right) .
\end{aligned}
$$

This implies that

$$
\left\|u_{n}-p\right\|^{2} \leq\left\|x_{n}-p\right\|^{2}-\left\|u_{n}-x_{n}\right\|^{2} .
$$

Then from (29) and (44), we derive that

$$
\begin{aligned}
&\left\|x_{n+1}-p\right\|^{2}\left\|\alpha_{n} \gamma f\left(x_{n}\right)+\left(\left(1-\beta_{n}\right) I-\alpha_{n} \mu F\right) u_{n}+\beta_{n} x_{n}-p\right\|^{2} \\
& \leq\left(\alpha_{n}\left\|\gamma f\left(x_{n}\right)-\mu F(p)\right\|+\beta_{n}\left\|x_{n}-p\right\|\right. \\
&\left.+\left\|\left(\left(1-\beta_{n}\right) I-\alpha_{n} \mu F\right) u_{n}-\left(\left(1-\beta_{n}\right) I-\alpha_{n} \mu F\right) p\right\|\right)^{2} \\
& \leq\left(\alpha_{n} \gamma\left\|f\left(x_{n}\right)-f(p)\right\|+\alpha_{n}\|\gamma f(p)-\mu F(p)\|\right. \\
&\left.+\left(1-\beta_{n}-\alpha_{n} \tau\right)\left\|u_{n}-p\right\|+\beta_{n}\left\|x_{n}-p\right\|\right)^{2} \\
& \leq\left(\left(\alpha_{n} \gamma \rho+\beta_{n}\right)\left\|x_{n}-p\right\|+\left(1-\beta_{n}-\alpha_{n} \tau\right)\left\|u_{n}-p\right\|\right. \\
&\left.+\alpha_{n}\|\gamma f(p)-\mu F(p)\|\right)^{2} \\
& \leq\left(\left(\alpha_{n} \tau+\beta_{n}\right)\left\|x_{n}-p\right\|+\left(1-\beta_{n}-\alpha_{n} \tau\right)\left\|u_{n}-p\right\|\right. \\
&\left.+\alpha_{n}\|\gamma f(p)-\mu F(p)\|\right)^{2} \\
& \leq\left(\alpha_{n} \tau+\beta_{n}\right)\left\|x_{n}-p\right\|^{2}+\left(1-\beta_{n}-\alpha_{n} \tau\right)\left\|u_{n}-p\right\|^{2} \\
&+\alpha_{n}^{2}\|\gamma f(p)-\mu F(p)\|^{2} \\
&+2\left[\left(\alpha_{n} \tau+\beta_{n}\right)\left\|x_{n}-p\right\|+\left(1-\beta_{n}-\alpha_{n} \tau\right)\left\|u_{n}-p\right\|\right] \\
& \times \\
& \alpha_{n}\|\gamma f(p)-\mu F(p)\| \\
& \leq\left(\alpha_{n} \tau+\beta_{n}\right)\left\|x_{n}-p\right\|^{2}+\left(1-\beta_{n}-\alpha_{n} \tau\right) \\
& \times\left(\left\|x_{n}-p\right\|^{2}-\left\|u_{n}-x_{n}\right\|^{2}\right)+\alpha_{n}^{2}\|\gamma f(p)-\mu F(p)\|^{2} \\
&+2\left\|x_{n}-p\right\| \alpha_{n}\|\gamma f(p)-\mu F(p)\|
\end{aligned}
$$




$$
\begin{aligned}
= & \left\|x_{n}-p\right\|^{2}-\left(1-\beta_{n}-\alpha_{n} \tau\right)\left\|u_{n}-x_{n}\right\|^{2} \\
& +\alpha_{n}^{2}\|\gamma f(p)-\mu F(p)\|^{2}+2\left\|x_{n}-p\right\| \alpha_{n}\|\gamma f(p)-\mu F(p)\| \\
\leq & \left(\left\|x_{n}-x_{n+1}\right\|+\left\|x_{n+1}-p\right\|\right)^{2}-\left(1-\beta_{n}-\alpha_{n} \tau\right)\left\|u_{n}-x_{n}\right\|^{2} \\
& +\alpha_{n}^{2}\|\gamma f(p)-\mu F(p)\|^{2}+2\left\|x_{n}-p\right\| \alpha_{n}\|\gamma f(p)-\mu F(p)\| \\
= & \left\|x_{n}-x_{n+1}\right\|^{2}+\left\|x_{n+1}-p\right\|^{2}+2\left\|x_{n}-x_{n+1}\right\|\left\|x_{n+1}-p\right\| \\
& -\left(1-\beta_{n}-\alpha_{n} \tau\right)\left\|u_{n}-x_{n}\right\|^{2}+\alpha_{n}^{2}\|\gamma f(p)-\mu F(p)\|^{2} \\
& +2\left\|x_{n}-p\right\| \alpha_{n}\|\gamma f(p)-\mu F(p)\| .
\end{aligned}
$$

Thus,

$$
\begin{aligned}
(1- & \left.\beta_{n}-\alpha_{n} \tau\right)\left\|u_{n}-x_{n}\right\|^{2} \\
\leq & \left\|x_{n}-x_{n+1}\right\|^{2}+2\left\|x_{n}-x_{n+1}\right\|\left\|x_{n+1}-p\right\| \\
& +\alpha_{n}^{2}\|\gamma f(p)-\mu F(p)\|^{2} \\
& +2\left\|x_{n}-p\right\| \alpha_{n}\|\gamma f(p)-\mu F(p)\| .
\end{aligned}
$$

From (41) and condition (C1), we obtain that

$$
\left(1-\beta_{n}-\alpha_{n} \tau\right)\left\|u_{n}-x_{n}\right\|^{2} \longrightarrow 0, \quad n \longrightarrow \infty .
$$

From conditions (C1) and (C3), we get that

$$
\lim _{n \rightarrow \infty}\left\|u_{n}-x_{n}\right\|=0
$$

Next, we show that

$$
\limsup _{n \rightarrow \infty}\left\langle(\gamma f-\mu F) z, x_{n}-z\right\rangle \leq 0,
$$

where $z \in S$ which solves the variational inequality

$$
\langle(\mu F-\gamma f) z, z-x\rangle \leq 0, \quad \forall x \in S .
$$

Since $\left\{x_{n}\right\}$ is bounded, without loss of generality, we may assume that $x_{n_{i}} \rightarrow \widetilde{z}$ such that

$$
\begin{aligned}
& \limsup _{n \rightarrow \infty}\left\langle(\gamma f-\mu F) z, x_{n}-z\right\rangle \\
& \quad=\lim _{i \rightarrow \infty}\left\langle(\gamma f-\mu F) z, x_{n_{i}}-z\right\rangle .
\end{aligned}
$$

From $\left\|u_{n}-x_{n}\right\| \rightarrow 0$ and $x_{n_{i}} \rightarrow \widetilde{z}$, we obtain that $u_{n_{i}} \rightarrow \widetilde{z}$.

Next, we show that $\widetilde{z} \in S=\operatorname{EP}\left(F_{1}\right)$.

Indeed, from $u_{n}=T_{r_{n}} x_{n}$, for any $y \in C$, we obtain

$$
F_{1}\left(u_{n}, y\right)+\frac{1}{r_{n}}\left\langle y-u_{n}, u_{n}-x_{n}\right\rangle \geq 0 .
$$

From (A2), we have

$$
\frac{1}{r_{n}}\left\langle y-u_{n}, u_{n}-x_{n}\right\rangle \geq F_{1}\left(y, u_{n}\right)
$$

and hence

$$
\left\langle y-u_{n_{i}}, \frac{u_{n_{i}}-x_{n_{i}}}{r_{n_{i}}}\right\rangle \geq F_{1}\left(y, u_{n_{i}}\right) .
$$

Since $\left(u_{n_{i}}-x_{n_{i}}\right) / r_{n_{i}} \rightarrow 0$ and $u_{n_{i}} \rightarrow \widetilde{z}$, it follows from (A4) that $F_{1}(y, \tilde{z}) \leq 0$, for any $y \in C$. Let $y_{t}=t y+(1-t) \widetilde{z}$, for all $t \in(0,1], y \in C$. Since $\left\{u_{n_{i}}\right\} \subset C, u_{n_{i}} \rightarrow \widetilde{z}$, and $C$ is closed and convex set, then we get $\widetilde{z} \in C$. Since $y \in C$ and $\widetilde{z} \in C$, then we have $y_{t} \in C$. Hence we have $F_{1}\left(y_{t}, \widetilde{z}\right) \leq 0$.

Thus, from (A1) and (A4), we have

$$
\begin{aligned}
0 & =F_{1}\left(y_{t}, y_{t}\right) \\
& =F_{1}\left(y_{t}, t y+(1-t) \tilde{z}\right) \\
& \leq t F_{1}\left(y_{t}, y\right)+(1-t) F_{1}\left(y_{t}, \widetilde{z}\right) \\
& \leq t F_{1}\left(y_{t}, y\right),
\end{aligned}
$$

and hence $F_{1}\left(y_{t}, y\right) \geq 0$. From (A3), we have $F_{1}(\widetilde{z}, y) \geq 0$, for any $y \in C$. Hence $\widetilde{z} \in \operatorname{EP}\left(F_{1}\right)=S$.

Therefore,

$$
\begin{aligned}
\limsup _{n \rightarrow \infty}\left\langle(\gamma f-\mu F) z, x_{n}-z\right\rangle & =\lim _{i \rightarrow \infty}\left\langle(\gamma f-\mu F) z, x_{n_{i}}-z\right\rangle \\
& =\langle(\gamma f-\mu F) z, \widetilde{z}-z\rangle \leq 0 .
\end{aligned}
$$

Finally, we show that $x_{n} \rightarrow z$.

As a matter of fact,

$$
\begin{aligned}
x_{n+1}-z= & \alpha_{n} \gamma f\left(x_{n}\right)+\left(\left(1-\beta_{n}\right) I-\alpha_{n} \mu F\right) u_{n}+\beta_{n} x_{n}-z \\
= & \alpha_{n} \gamma f\left(x_{n}\right)-\alpha_{n} \mu F(z)+\beta_{n} x_{n}-\beta_{n}(z) \\
& +\left(\left(1-\beta_{n}\right) I-\alpha_{n} \mu F\right) u_{n}-\left(\left(1-\beta_{n}\right) I-\alpha_{n} \mu F\right) z \\
= & \alpha_{n} \gamma\left(f\left(x_{n}\right)-f(z)\right) \\
& +\alpha_{n}(\gamma f(z)-\mu F(z))+\beta_{n}\left(x_{n}-z\right) \\
& +\left(\left(1-\beta_{n}\right) I-\alpha_{n} \mu F\right) u_{n}-\left(\left(1-\beta_{n}\right) I-\alpha_{n} \mu F\right) z .
\end{aligned}
$$

So, from (29), we derive

$$
\begin{aligned}
& \left\|x_{n+1}-z\right\|^{2} \\
& =\alpha_{n} \gamma\left\langle f\left(x_{n}\right)-f(z), x_{n+1}-z\right\rangle \\
& \quad+\left\langle\left(\left(1-\beta_{n}\right) I-\alpha_{n} \mu F\right) u_{n}\right. \\
& \left.\quad \quad\left(\left(1-\beta_{n}\right) I-\alpha_{n} \mu F\right) z, x_{n+1}-z\right\rangle \\
& \quad+\alpha_{n}\left\langle\gamma f(z)-\mu F(z), x_{n+1}-z\right\rangle+\beta_{n}\left\langle x_{n}-z, x_{n+1}-z\right\rangle \\
& \leq \alpha_{n} \gamma \rho\left\|x_{n}-z\right\| \cdot\left\|x_{n+1}-z\right\| \\
& \quad+\left(1-\beta_{n}-\alpha_{n} \tau\right)\left\|u_{n}-z\right\| \cdot\left\|x_{n+1}-z\right\| \\
& \quad+\alpha_{n}\left\langle\gamma f(z)-\mu F(z), x_{n+1}-z\right\rangle \\
& +\beta_{n}\left\|x_{n}-z\right\| \cdot\left\|x_{n+1}-z\right\|
\end{aligned}
$$




$$
\begin{aligned}
\leq & \left(1-\alpha_{n}(\tau-\gamma \rho)\right)\left\|x_{n}-z\right\| \cdot\left\|x_{n+1}-z\right\| \\
& +\alpha_{n}\left\langle\gamma f(z)-\mu F(z), x_{n+1}-z\right\rangle \\
\leq & \frac{1-\alpha_{n}(\tau-\gamma \rho)}{2}\left(\left\|x_{n}-z\right\|^{2}+\left\|x_{n+1}-z\right\|^{2}\right) \\
& +\alpha_{n}\left\langle\gamma f(z)-\mu F(z), x_{n+1}-z\right\rangle .
\end{aligned}
$$

It follows that

$$
\begin{aligned}
&\left\|x_{n+1}-z\right\|^{2} \\
& \leq \frac{1-\alpha_{n}(\tau-\gamma \rho)}{1+\alpha_{n}(\tau-\gamma \rho)}\left\|x_{n}-z\right\|^{2} \\
&+\frac{2 \alpha_{n}}{1+\alpha_{n}(\tau-\gamma \rho)}\left\langle\gamma f(z)-\mu F(z), x_{n+1}-z\right\rangle \\
& \leq\left(1-\alpha_{n}(\tau-\gamma \rho)\right)\left\|x_{n}-z\right\|^{2} \\
&+\frac{2 \alpha_{n}}{1+\alpha_{n}(\tau-\gamma \rho)}\left\langle\gamma f(z)-\mu F(z), x_{n+1}-z\right\rangle .
\end{aligned}
$$

Hence,

$$
\left\|x_{n+1}-z\right\|^{2} \leq\left(1-\gamma_{n}\right)\left\|x_{n}-z\right\|^{2}+\gamma_{n} \delta_{n}
$$

where $\gamma_{n}=\alpha_{n}(\tau-\gamma \rho), \gamma_{n} \delta_{n}=\left(2 \alpha_{n} /\left(1+\alpha_{n}(\tau-\gamma \rho)\right)\right)\langle\gamma f(z)-$ $\left.\mu F(z), x_{n+1}-z\right\rangle$.

Since $\alpha_{n} \rightarrow 0$ and $\sum_{n=0}^{\infty} \alpha_{n}=\infty$, we get $\gamma_{n} \rightarrow 0$ and $\sum_{n=0}^{\infty} \gamma_{n}=\infty$. From (49), we get

$$
\begin{aligned}
\limsup _{n \rightarrow \infty} \delta_{n}=\limsup _{n \rightarrow \infty} & \frac{2}{\left(1+\alpha_{n}(\tau-\gamma \rho)\right)(\tau-\gamma \rho)} \\
& \times\left\langle\gamma f(z)-\mu F(z), x_{n+1}-z\right\rangle \leq 0 .
\end{aligned}
$$

Now, applying Lemma 6 to (60) concludes that $x_{n} \rightarrow z$ as $n \rightarrow \infty$.

Remark 9. Our proposed algorithm (23) is more general than $\mathrm{Su}$ and Li's algorithm (5), because of introducing nonlinear operator $F$.

If $\gamma=1, \mu=1$, and $F=I$, we obtain the following Corollary.

Corollary 10. Let $H$ be a real Hilbert space and let $C$ be a nonempty, closed, and convex subset of $H$. Let $h: C \rightarrow \mathbb{R}$ be a real-valued convex function. Assume that $h(x)$ is lower semicontinuous. Let $f: H \rightarrow H$ be a contraction with a coefficient $0<\rho<1$. Suppose that the optimization problem (3) is consistent and let $S$ denote its solution set. Let $\left\{x_{n}\right\}_{n=0}^{\infty}$ be generated by $x_{1} \in H$ and

$$
\begin{aligned}
& h(y)-h\left(u_{n}\right)+\frac{1}{r_{n}}\left\langle y-u_{n}, u_{n}-x_{n}\right\rangle \geq 0, \quad \forall y \in C, \\
& x_{n+1}=\alpha_{n} f\left(x_{n}\right)+\left(1-\beta_{n}-\alpha_{n}\right) u_{n}+\beta_{n} x_{n}, \quad n \in \mathbb{N},
\end{aligned}
$$

where $u_{n}=T_{r_{n}} x_{n}$. Let $\left\{\alpha_{n}\right\},\left\{\beta_{n}\right\} \subset(0,1),\left\{r_{n}\right\} \subset(0, \infty)$ satisfy the following conditions:

(C1) $\lim _{n \rightarrow \infty} \alpha_{n}=0$ and $\sum_{n=1}^{\infty} \alpha_{n}=\infty$;

(C2) $\sum_{n=1}^{\infty}\left|\alpha_{n+1}-\alpha_{n}\right|<\infty$;

(C3) $\lim \sup _{n \rightarrow \infty} \beta_{n}<1$ and $\sum_{n=1}^{\infty}\left|\beta_{n+1}-\beta_{n}\right|<\infty$;

(C4) $\liminf _{n \rightarrow \infty} r_{n}>0$ and $\sum_{n=1}^{\infty}\left|r_{n+1}-r_{n}\right|<\infty$.

Then the sequence $\left\{x_{n}\right\}$ generated by (62) converges strongly to a point $z \in S$, which solves the variational inequality

$$
\langle(I-f) z, z-x\rangle \leq 0, \quad \forall x \in S .
$$

Remark 11. We would like to point out that the conditions of algorithm (62) are different from those of algorithm (4.3) in [2]. The conditions of the sequence $\left\{\alpha_{n}\right\}$ and the sequence $\left\{r_{n}\right\}$ of parameters in (4.3) are the same as the ones of algorithm (62), but the conditions of the sequence $\left\{\beta_{n}\right\}$ of parameters in (4.3) are $\beta_{n}=o\left(\alpha_{n}\right)$ and $\sum_{n=1}^{\infty}\left|\beta_{n+1}-\beta_{n}\right|<\infty$. It is obvious that our conditions are much weaker.

\section{Application}

In this section, we give an application of Theorem 8 to the split feasibility problem (say SFP, for short) which was introduced by Censor and Elfving [15]. Since its inception in 1994, the split feasibility problem (SFP) has received much attention (see [16-18]) due to its applications to signal processing and image reconstruction, with particular progress in intensitymodulated radiation therapy.

The SFP can mathematically be formulated as the problem of finding a point $x$ with the property

$$
x \in C, \quad B x \in Q \text {, }
$$

where $C$ and $Q$ are nonempty closed convex subsets of Hilbert spaces $H_{1}$ and $H_{2}$, respectively. $B: H_{1} \rightarrow H_{2}$ is a bounded linear operator.

It is clear that $x^{*}$ is a solution to the split feasibility problem (64) if and only if $x^{*} \in C$ and $B x^{*}-P_{Q} B x^{*}=0$. We define the proximity function $h$ by

$$
h(x)=\frac{1}{2}\left\|B x-P_{Q} B x\right\|^{2}
$$

and consider the following optimization problem:

$$
\min _{x \in C} h(x)=\min _{x \in C} \frac{1}{2}\left\|B x-P_{\mathrm{Q}} B x\right\|^{2} .
$$

Then, $x^{*}$ solves the split feasibility problem (64) if and only if $x^{*}$ solves the minimization problem (66) with the minimization being equal to 0 . Byrne [19] introduced the socalled CQ algorithm to solve the SFP:

$$
x_{n+1}=P_{C}\left(I-\gamma B^{*}\left(I-P_{\mathrm{Q}}\right) B\right) x_{n}, \quad n \geq 0,
$$

where $0<\gamma<2 /\|B\|^{2}$. He obtained that the sequence $\left\{x_{n}\right\}$ generated by (67) converges weakly to a solution of the SFP. 
In order to obtain strong convergence iterative sequence to solve the SFP (64), we propose the following iterative algorithm by $x_{1} \in H_{1}$ and

$$
\begin{aligned}
& \frac{1}{2}\left\|B y-P_{\mathrm{Q}} B y\right\|^{2}-\frac{1}{2}\left\|B u_{n}-P_{\mathrm{Q}} B u_{n}\right\|^{2} \\
& +\frac{1}{r_{n}}\left\langle y-u_{n}, u_{n}-x_{n}\right\rangle \geq 0, \quad \forall y \in C, \\
& x_{n+1}=\alpha_{n} \gamma f\left(x_{n}\right)+\left(\left(1-\beta_{n}\right) I-\alpha_{n} \mu F\right) u_{n}+\beta_{n} x_{n}, \\
& n \in \mathbb{N},
\end{aligned}
$$

where $u_{n}=T_{r_{n}} x_{n}$ and $T_{r_{n}}: H_{1} \rightarrow C$ is a mapping defined as in Lemma 5, where $f: H_{1} \rightarrow H_{1}$ is a contraction with a coefficient $0<\rho<1$ and $F: C \rightarrow H_{1}$ is a $k$-Lipschitzian and $\eta$-strongly monotone operator with constants $k>0, \eta>0$. Suppose that $0<\mu<2 \eta / k^{2}, 0<\gamma<\tau / \rho$, where $\tau=\mu \eta-$ $\left(\mu^{2} k^{2} / 2\right)$. We can show that the sequence $\left\{x_{n}\right\}$ generated by (68) converges strongly to a solution of the SFP (64), if the sequence $\left\{\alpha_{n}\right\},\left\{\beta_{n}\right\} \subset(0,1)$ and the sequence $\left\{r_{n}\right\} \subset(0, \infty)$ of parameters satisfy appropriate conditions.

Applying Theorem 8, we obtain the following result.

Theorem 12. Assume that the split feasibility problem (64) is consistent. Let the sequence $\left\{x_{n}\right\}$ be generated by (68), where the sequence $\left\{\alpha_{n}\right\},\left\{\beta_{n}\right\} \subset(0,1)$ and the sequence $\left\{r_{n}\right\} \subset$ $(0, \infty)$ satisfy the conditions $(C 1)-(C 5)$. Then the sequence $\left\{x_{n}\right\}$ converges strongly to a solution of the split feasibility problem (64).

Proof. We define the proximity function $h$ by

$$
h(x)=\frac{1}{2}\left\|B x-P_{\mathrm{Q}} B x\right\|^{2}
$$

and consider the following optimization problem:

$$
\min _{x \in C} h(x)=\min _{x \in C} \frac{1}{2}\left\|B x-P_{Q} B x\right\|^{2},
$$

where $h: C \rightarrow \mathbb{R}$ is a real-valued convex function and $h(x)$ is lower semicontinuous. Then, $x^{*}$ solves the split feasibility problem (64) if and only if $x^{*}$ solves the minimization problem (66) with the minimization being equal to 0 .

Consequently, the iterative scheme (68) is equivalent to

$$
\begin{gathered}
x_{1} \in H_{1}, \\
h(y)-h\left(u_{n}\right)+\frac{1}{r_{n}}\left\langle y-u_{n}, u_{n}-x_{n}\right\rangle \geq 0, \quad \forall y \in C, \\
x_{n+1}=\alpha_{n} \gamma f\left(x_{n}\right)+\left(\left(1-\beta_{n}\right) I-\alpha_{n} \mu F\right) u_{n}+\beta_{n} x_{n}, \quad n \in \mathbb{N},
\end{gathered}
$$

where $u_{n}=T_{r_{n}} x_{n}$ and $T_{r_{n}}: H_{1} \rightarrow C$ is a mapping defined as in Lemma 5. Due to Theorem 8, we have the conclusion immediately.

\section{Conflict of Interests}

The authors declare that there is no conflict of interests regarding the publication of this paper.

\section{Acknowledgments}

The authors would like to thank the referees for their helpful comments, which notably improved the presentation of this paper. This work was completed with the support of the Fundamental Research Funds for the Central Universities (no. 3122013D017).

\section{References}

[1] W. Takahashi, Nonlinear Functional Analysis, Yokohama Publishers, Yokohama, Japan, 2000.

[2] Y. F. Su and M. Q. Li, "Approximation of solutions of equilibrium problems and application in optimization problems," Journal of Applied Mathematics and Mechanics, vol. 6, pp. 82-93, 2010.

[3] I. Yamada, "The hybrid steepest descent for the variational inequality problems over the intersection of fixed points sets of nonexpansive mapping," in Inherently Parallel Algorithms in Feasibility and Optimization and Their Application, D. Butnariu, Y. Censor, and S. Reich, Eds., pp. 473-504, Elservier, New York, NY, USA, 2001.

[4] G. Marino and H.-K. Xu, "A general iterative method for nonexpansive mappings in Hilbert spaces," Journal of Mathematical Analysis and Applications, vol. 318, no. 1, pp. 43-52, 2006.

[5] M. Tian, "A general iterative algorithm for nonexpansive mappings in Hilbert spaces," Nonlinear Analysis: Theory, Methods \& Applications, vol. 73, no. 3, pp. 689-694, 2010.

[6] J. S. Jung, "Some results on a general iterative method for $k$ strictly pseudo-contractive mappings," Fixed Point Theory and Applications, vol. 2011, article 24, 2011.

[7] H. Brézis, Opérateurs Maximaux Monotones et Semi-Groupes de Contractions dans les Espaces de Hilbert, North-Holland, Amsterdam, The Netherlands, 1973.

[8] C. Jaiboon and P. Kumam, "A hybrid extragradient viscosity approximation method for solving equilibrium problems and fixed point problems of infinitely many nonexpansive mappings," Fixed Point Theory and Applications, vol. 2009, Article ID 374815, 2009.

[9] T. Jitpeera, P. Katchang, and P. Kumam, "A viscosity of Cesàro mean approximation methods for a mixed equilibrium, variational inequalities, and fixed point problems," Fixed Point Theory and Applications, vol. 2011, Article ID 945051, 2011.

[10] D. P. Bertsekas and E. M. Gafni, "Projection methods for variational inequalities with application to the traffic assignment problem," Mathematical Programming Study, no. 17, pp. 139-159, 1982.

[11] D. Han and H. K. Lo, "Solving non-additive traffic assignment problems: a descent method for co-coercive variational inequalities," European Journal of Operational Research, vol. 159, no. 3, pp. 529-544, 2004.

[12] E. Blum and W. Oettli, "From optimization and variational inequalities to equilibrium problems," The Mathematics Student, vol. 63, no. 1-4, pp. 123-145, 1994.

[13] S. D. Flåm and A. S. Antipin, "Equilibrium programming using proximal-like algorithms," Mathematical Programming, vol. 78, no. 1, pp. 29-41, 1997.

[14] H.-K. Xu, "Iterative algorithms for nonlinear operators," Journal of the London Mathematical Society. Second Series, vol. 66, no. 1, pp. 240-256, 2002.

[15] Y. Censor and T. Elfving, "A multiprojection algorithm using Bregman projections in a product space," Numerical Algorithms, vol. 8, no. 2-4, pp. 221-239, 1994. 
[16] G. López, V. Martín-Márquez, F. Wang, and H.-K. Xu, “Solving the split feasibility problem without prior knowledge of matrix norms," Inverse Problems, vol. 28, no. 8, p. 18, article 085004, 2012.

[17] J. Zhao, Y. Zhang, and Q. Yang, "Modified projection methods for the split feasibility problem and the multiple-sets split feasibility problem," Applied Mathematics and Computation, vol. 219, no. 4, pp. 1644-1653, 2012.

[18] H.-K. Xu, "Iterative methods for the split feasibility problem in infinite-dimensional Hilbert spaces," Inverse Problems, vol. 26, no. 10, p. 17, article 105018, 2010.

[19] C. Byrne, "A unified treatment of some iterative algorithms in signal processing and image reconstruction," Inverse Problems, vol. 20, no. 1, pp. 103-120, 2004. 


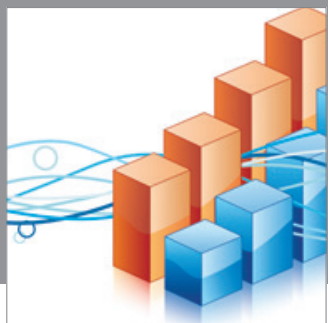

Advances in

Operations Research

mansans

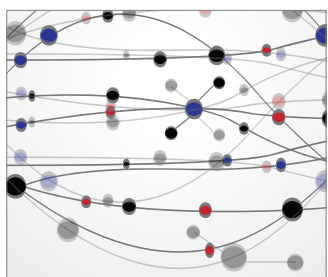

The Scientific World Journal
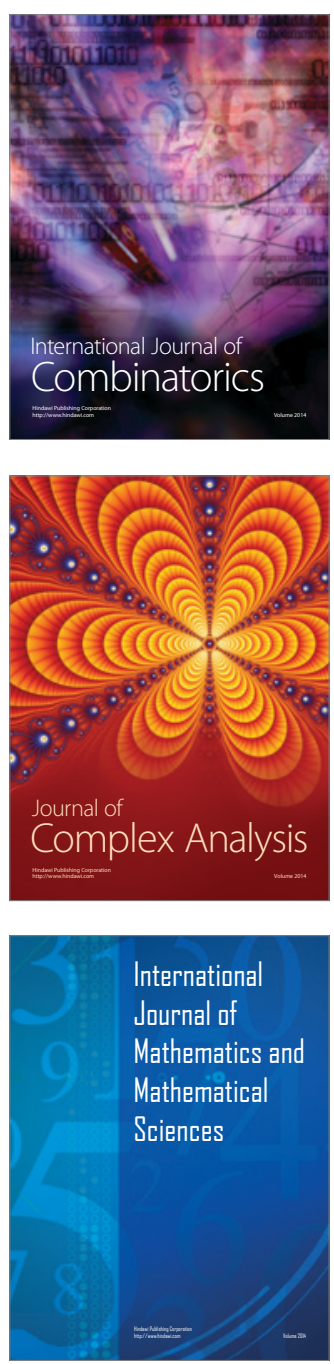
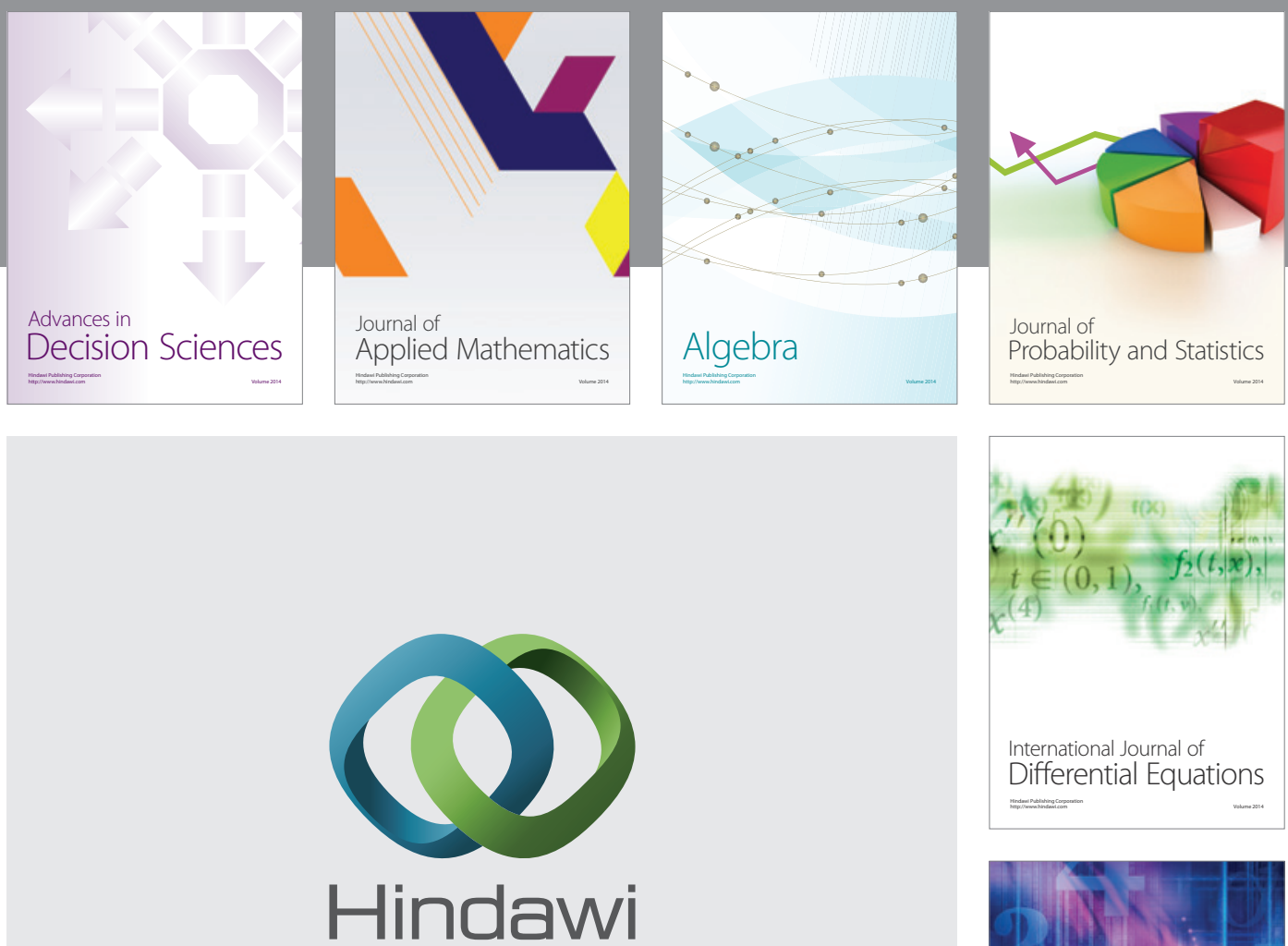

Submit your manuscripts at http://www.hindawi.com
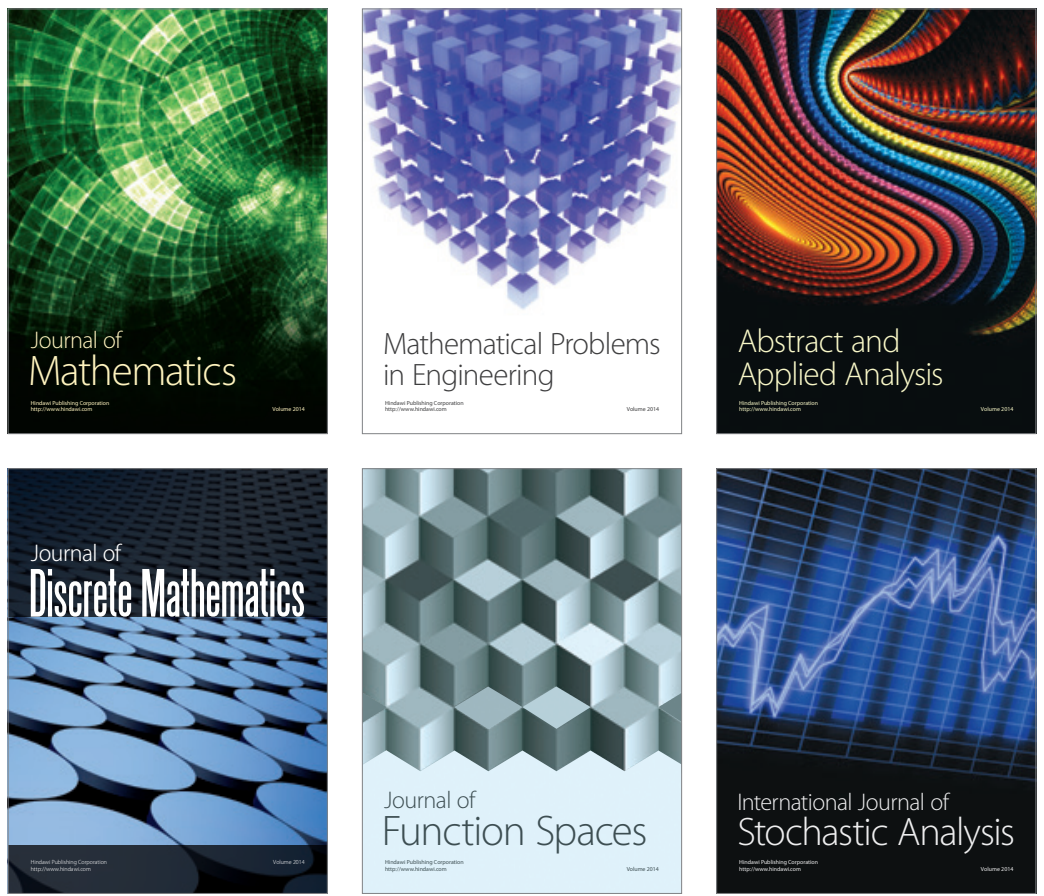

Journal of

Function Spaces

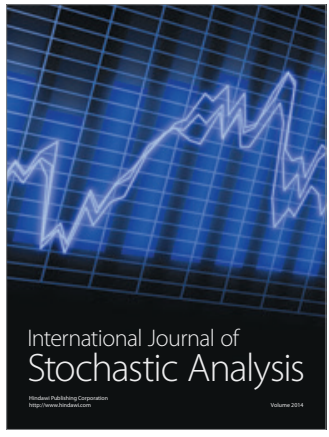

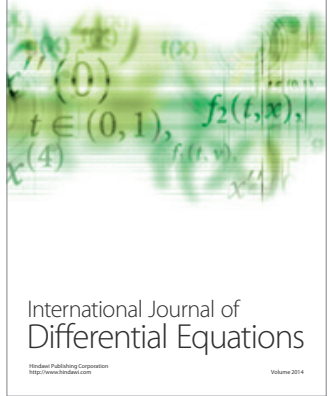
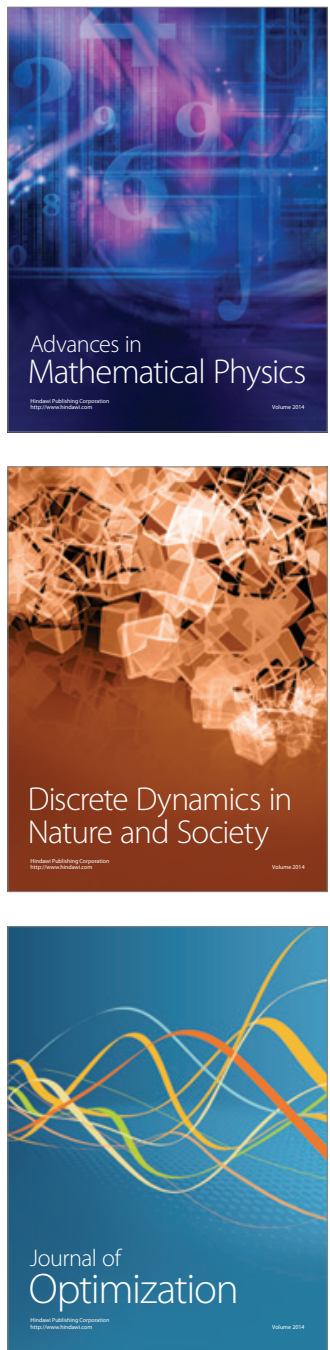